\title{
МОДЕРНИЗИРОВАННЫЙ МЕТОД КОНТРОЛЯ ГРУПП КВАДРОКОПТЕРОВ
}

\section{THE UPDATED METHOD OF CONTROL OF GROUP OF QUADROCOPTERS}

ФГАОУ ВО «Северо-Кавказский федеральный университет» г. Ставрополь / FSAEI HE "North-Caucasus Federal University"

\begin{abstract}
Аниотация: в статье представлен метод управления роем квадрокоптеров. Для координации группь необходимо формировать пространственную програминую траекторию БПЛА с помощью соответствующего закона управления. Вводится понятие координированного разворота, который позволяет получить аналитические уравнения пространственных движений, выраженных через определение вектора скоростей и угла рыскания. Метод был протестирован в симуляmоре Gazebo. Результатьи используются для пространственных перемещений групп квадрокоптеров.
\end{abstract}

Ключевые слова: групповое управление, контроль формаций, роевое движение, квадрокоптер, БПЛА.

Abstract. In this article, the method of swarm control was presented. To coordinate the group, the spatial trajectory of $U A V$ has to be implemented by applying efficient control law. The principle of coordinated rotation has been introduced. The coordinated rotation allows receiving analytical equations of spatial motion which are represented within defining of the vector of velocity and yaw angle. The method was evaluated in Gazebo simulator. All results were utilized to move the group within the environment.

Key words: group control, the control of formation, swarm motion, quadrocopter, UAV.

Введение. За последние 10 лет наблюдается широкое и массовое использование БПЛА для решения практических задач [4]. В настоящее время, рынок беспилотных технологий предлагает огромный выбор доступных по цене и простых в сборке моделей БПЛА с мощными бортовыми вычислителями и возможностью установки дополнительных устройств. Нанболее популярным типом является квадрокоптер, используемый как в коммерческой индустрии, так и в академической среде. Квадрокоптеры оборудованы бортовыми сенсорами и популярны из-за своего размера и наличием возможности “зависать” над объектами и свободно перемещаться в пространстве. Квадрокоптеры могут быть использованы в различных областях, начиная от мониторинга, задействования их в поисковых мероприятиях до применения в спасательных операциях [4]. Квадрокоптеры объединенные в группы могут покрыть большее пространство, собрать большее количество информации и менее восприимчивы к потерям участников группы.

Материалы и методы. Обзор известных методов решения групповой задачи. Групповой контроль может быть выполнен с помощьо использования нескольких различных стратегий [9]. Каждая из них имеет преимушества и недостатки, в зависимости от выполнясмой задачи. На сегодняшний день в мире существует ряд примеров демонстриругщих работоспособность алгоритмов группового управления.

Все используемые алгоритмы группового контроля можно разделить на три типа: лидер-следователь [1], виртуальную структуру [2] и поведенческий подход [3]. В первом типе один из участников выбирается как лидер группы, остальные корректируют свое движение согласно лидеру. Преимушество такого метода заключастся в том, что упрошается контроль группы, поскольку управление осуществляется только лидером. Однако в случае выхода из строя лидера, контроль всей групюй будет потерян. Виртуальная структура рассматривает всю группу как фиксированную структуру, представленную как одна контрольная точка. Поведенческий подход представляет запрограммированное движение группы для выполнения определенных действий, уход от коллизий, движение группы с сохранением максимально возможного малого расстояния между участниками и Т.Д.

В большинстве случаев этот тип групповой стратегии основан на реальных природшьх феноменах. Для движения группы, в данюй статье предлагается использовать метод, базиругщийся на стратегии виртуального лидера.

\section{Результаты и обсуждения}

\section{1. Математическая моде.ль движения квадрокоптера}

Для начала опишем систему квадрокоптера, которая имеет 6 стененей свободы и представлена 12-го параметрами, такими как углы Эйлера $\varphi \Psi \theta[2]$. Применение углов Эйлера в системе координат, связанной с землей обозначается как p, q, r. Остальные параметры являотся позициями в паземной системе координат х, у, z. Скорости представлены в локальной системе координат $\mathrm{U}, \boldsymbol{\omega}$.

Далее опишем Матрицу поворота [6] вокруг трех осей.

Поворот вокруг оси ОХ описывается матрицей: 
вокруг оси OY:

$$
\mathrm{R}_{\mathrm{x}}=\left(\begin{array}{ccc}
1 & 0 & 0 \\
0 & \cos \vartheta & \sin \vartheta \\
0 & -\sin \vartheta & \cos \vartheta
\end{array}\right)
$$

вогруг оси OZ:

$$
\mathrm{R}_{\mathrm{y}}=\left(\begin{array}{ccc}
\cos \theta & 0 & -\sin \theta \\
0 & 1 & 0 \\
\sin \theta & 0 & \cos \theta
\end{array}\right)
$$

$$
\mathrm{R}_{\mathrm{z}}=\left(\begin{array}{ccc}
\cos \Psi & \sin \Psi & 0 \\
-\sin \Psi & \cos \Psi & 0 \\
0 & 0 & 1
\end{array}\right)
$$

Матрица $\mathrm{D}=\mathrm{R}_{\mathrm{x}} * \mathrm{R}_{\mathrm{y}}$ * $\mathrm{R}_{\mathrm{z}}$ описывает перевод из глобальной системы координат в локальную [5]. Это матрица представлена как:

$$
\mathrm{D}=\left(\begin{array}{ccc}
\cos \theta \cos \psi & \cos \theta \sin \psi & -\sin \theta \\
\sin \vartheta \sin \theta \cos \Psi-\cos \varphi \sin \psi & \sin \vartheta \sin \theta \sin \psi+\cos \vartheta \cos \Psi & \sin \vartheta \cos \theta \\
\cos \varphi \sin \theta \cos \Psi+\sin \vartheta \sin \vartheta & \cos \vartheta \sin \theta \sin \psi-\sin \vartheta \cos \vartheta & \cos \vartheta \cos \theta
\end{array}\right) .
$$

Трансформация от скоростей в глобальной системе координат к скоростям в локальной системе координат определяется выражениями:

$$
\left(\begin{array}{c}
\dot{x} \\
\dot{y} \\
\dot{z}
\end{array}\right)=\mathrm{D}^{-1}\left(\begin{array}{l}
u \\
v \\
\omega
\end{array}\right)
$$

Трансформация угловых скоростей для перевода из одной системы координат в другую определяется как:

где Е-матрица представлена:

$$
\left(\begin{array}{c}
p \\
q \\
r
\end{array}\right)=\mathrm{E}\left(\begin{array}{c}
\dot{\varphi} \\
\dot{\theta} \\
\dot{\psi}
\end{array}\right)
$$

$$
\mathrm{E}=\left(\begin{array}{ccc}
1 & 0 & -\sin \theta \\
0 & \cos \vartheta & \sin \vartheta \cos \theta \\
0 & -\sin \vartheta & \cos \vartheta \cos \theta
\end{array}\right)
$$
ординат [7]:

Далее опишем уравнение движения, представляюпее второй закон Ньютона в глобальной системе когде $\mathrm{m}$ - масса квадрокоптера, $\mathrm{V}$ - скорость квадрокоптера в глобальной системе координат. Записывая полпуго производную скорости по времени, перепишем закон в виде:

$$
\mathrm{F}=\mathrm{m} \dot{V}+\mathrm{m}(\vec{\omega} * \vec{V}),
$$

или

$$
\left(\begin{array}{c}
F_{x} \\
F_{y} \\
F_{z}
\end{array}\right)=m\left(\begin{array}{c}
\dot{u} \\
\dot{v} \\
\dot{\omega}
\end{array}\right)+m\left(\begin{array}{c}
p \\
q \\
r
\end{array}\right) *\left(\begin{array}{c}
u \\
v \\
\omega
\end{array}\right) .
$$

После выполнения векторного произведения, уравнение второго закона Ньютона будет записано как:

$$
\left(\begin{array}{l}
F_{x} \\
F_{y} \\
F_{z}
\end{array}\right)=m\left(\begin{array}{l}
\dot{u}+q \omega-r v \\
\dot{v}+r u-p \omega \\
\omega+p \dot{v}-q u
\end{array}\right) .
$$

Пренебрегая всеми силами, кроме тяги и гравитации уравнение выше перепишем как:

$$
\left(\begin{array}{c}
0 \\
0 \\
m g-T
\end{array}\right)=m\left(\begin{array}{l}
\dot{u}+q \omega-r v \\
\dot{v}+r u-p \omega \\
\omega+p \dot{v}-q u
\end{array}\right) .
$$

Далее осуществляется переход к локальной системе координат с помощью трансферной матрицы $\mathrm{D}$, получаем систему уравнсний:

$$
\begin{gathered}
\dot{u}=r v-q \omega-g \sin \Theta \\
\dot{v}=p \omega-r u+g \sin \Theta \sin \varphi \\
\dot{\omega}=q u-p v+g \cos \varphi \cos \theta-\frac{1}{m} .
\end{gathered}
$$

Затем представим уравнение тяги всех двигателей как

$$
T=b\left(\Omega_{1}^{2} \Omega_{2}^{2} \Omega_{3}^{2} \Omega_{4}^{2}\right)
$$

где b - коэффициент тяги, $\Omega$-угловая скорость каждого двигателя (i=1, 2, 3, 4).

В этом случае уравнепие 14 перепишется в следующем виде:

$$
\begin{gathered}
\dot{u}=r v-q \omega-g \sin \theta \\
\dot{v}=p \omega-r u+g \cos \theta * \sin \varphi
\end{gathered}
$$




$$
\dot{\omega}=q r-p v+g \cos \varphi \cos \theta-\frac{b}{m}\left(\Omega_{1}^{2}+\Omega_{2}^{2}+\Omega_{3}^{2}+\Omega_{4}^{2}\right) .
$$

Для представления полной математической модели движения квадрокоптера опишем врашательное движение [8].

Представим моменты вращения в виде следующего равенства:

$$
M=\dot{H} \text {. }
$$

Полную производную вектора Н запишем в виде:

Определим:

$$
M=\dot{H}+\omega * H \text {. }
$$

$$
\mathrm{H}=\mathrm{I}^{*}(\omega)
$$

где I - момент инерции, $\omega=(p, q, r)-$ вектор.

Следуюшим этапом запишем тензор момента инерции как:

$$
\mathrm{I}=\left(\begin{array}{ccc}
I_{x} & 0 & 0 \\
0 & I_{y} & 0 \\
0 & 0 & I_{y}
\end{array}\right) .
$$

Тогда уравнение 17 перепишем в следующую систему:

$$
\begin{gathered}
M=I \dot{\omega}+\omega * I \omega \\
M_{x}=\dot{p} I_{x}+q r\left(I_{z} I_{y}\right) \\
M_{y}=\dot{q} I_{y}+\operatorname{pr}\left(I_{x} I_{z}\right) \\
M_{z}=\dot{z} I_{z}+p q\left(I_{y} I_{x}\right) .
\end{gathered}
$$

Согласно симметрии, относительно плоскости XZ и $\mathrm{YZ}$ получим:

Упрощаем уравнение 20:

$$
\mathrm{I}_{\mathrm{x}}=\mathrm{I}_{\mathrm{y}} \text {. }
$$

Учитывая силу тяги и сопротивление, моменты будут переписаны как:

$$
\begin{gathered}
M_{x}=\dot{p} I_{x}+q r\left(I_{z}-I_{x}\right) \\
M_{y}=\dot{q} I_{y}+p r\left(I_{x}-I_{z}\right) \\
M_{z}=\dot{r} I_{z} . \\
M_{x}=l b\left(\Omega_{2}^{2} \Omega_{4}^{2}\right) \\
M_{y}=l b\left(\Omega_{1}^{2} \Omega_{3}^{2}\right) \\
M_{z}=d\left(\Omega_{2}^{2} \Omega_{4}^{2} \Omega_{1}^{2} \Omega_{3}^{2}\right),
\end{gathered}
$$

где $\mathrm{d}$ - коэффициент сопротивления, 1 - длина винта.

Преобразования системы 22 в уравнения движения представляем, как:

$$
\begin{gathered}
\dot{p}=\frac{l b}{I_{x}}\left(\Omega_{2}^{2} \Omega_{4}^{2}\right)-q r\left(\frac{I_{z}-I_{x}}{l_{x}}\right) \\
\dot{r}=\frac{a}{l_{z}}\left(\Omega_{2}^{2}+\Omega_{4}^{2}-\Omega_{1}^{2}-\Omega_{3}^{2}\right) .
\end{gathered}
$$$$
\dot{q}=\frac{l b}{l_{y}}\left(\Omega_{1}^{2} \Omega_{3}^{2}\right)-p r\left(\frac{l_{x}-l_{Z}}{l_{y}}\right)
$$

Следующим этапом введем описание динамики двигателя [6].

Tepa:

где $\mathrm{J}_{\mathrm{r}}$ - момент инерции вала

Уравнения Кирхгоффа и второй закон Ньютона представляют собой уравнения двигателей квадрокопе-

$$
\left\{\begin{array}{c}
J_{r} \ddot{\omega}_{m}+b \dot{\omega}_{m}=K_{t} i \\
L \frac{d_{i}}{d_{t}}+R_{i}=U-K_{e} \dot{\omega}_{m}
\end{array}\right.
$$

$\mathrm{b}$ - коэффициент вязкого трения

$\mathrm{K}_{\mathrm{e}}-$ коэффициент ЭДС

$\dot{K}_{t}-$ момент вращения двигателя

$\mathrm{R}$ - электрическое сопротивление

$\mathrm{L}$ - индуктивность.

\section{2. Описание конфигурации квадрокоптеров}

Для проведения практических экспериментов были выбраны два квадрокоптера Клевер, которые были произведены компанией Copter Express. Базовая конфигурация квадрокоптеров включала $2300 \mathrm{Kv}$ моторы, $30 \mathrm{~A}$ контроллеры оборотов скоростей (ESCs), четыре 5046 винта и 8 канальную станцию ручного управления на 2.4 Ггц. В качестве полетного контроллера использовался Pixracer r14 [10].

Данший полетный контроллер имеет следующие характеристики: процессор 180 Мгц ARM Cortex M4, память 256 Кб, ICM-20608 Акселерометр / Гироскоп (4 Ггц) / MPU9250 Акселерометр / Гиро / Магнитометр (4 Ггц), магнитометр с температурной компенсацией HMC5983, барометр MS5611, карта памяти типа тісгоSD для логгирования полетов. 
Полетный контроллер с установленным автопилотом РX4 обеспечивает отправку сигналов пाирокоимпульсной модуляции на 4 контроллера оборотов, контролирующих скорости вращения двигателя, а также его тягу. Сенсоры на борту включают гироскоп и акселерометр для определения скоростей и углового положения в пространстве. Отдельный GPS модуль используется для определения положения квадрокоптера относительно земли. Управление квадрокоптера осуществляется через LoRa 2.4 Ггц модуль, осуществляющий дуп.лексную передачу как управ.тяюших сигналов от наземной станции на квадрокоптер, так и телеметрии от квадрокоптера на наземную станцию.

Наземная станция представлена ноутбуком с установленной Linux орнентированной операционной системой с набором необходимых биб.пиотек.

\section{3. Программное обеспечение}

Приложение разработано под семейство систем Linux на языке программирования C++ 17 стандарта. Процесс проектирования происходил под Ubuntu 19.10, тестирование осушествлялось как на реальных БПЛА, так и в системе симуляции, состояшей из среды Gazebo, программной симуляции прошивки РХ4 и модуля MAVROS. Система симуляции запускается в docker-контейнере, чем обеспечивает переносимость и развёртываемость на других операционных системах, отличных от указанной.

ПО является модульным, что предполагает подгрузку каких-либо сериализованных команд, параметрических функций и моделей поведения роя. Управление централизованное, на данном этапе дроны не производят коммуникацию между собой и являются простыми исполнителями команд и отправляют телеметрию на наземную станцию. Для коммуникации с реальными квадрокоптерами используется библиотека MavSDK. Помимо подключения к дронам, использующим Mavlink, возможно создание простых виртуальных объектов в симуляции, обладаюших инерцией, для докомплектования оригинального роя виртуальными участниками.

В качестве точки отсчёта для определения позиции группы используется геодезическая координата первого подключённого к приложению квадрокоптера

В основе алгоритма лежит стратегия виртуального лидера. Каждый агент группы сохраняет фиксированное расстояние относительно лидера.

Управление роем осуществляется путём задания компонентов системы: метода построения роя и стратегии перемещения всего роя. Метод построения роя может также содержать набор команд для ввода, например, перемещение отдельного элемента роя. Стратегия перемещения представляет собой набор вводимых команд, которые так или иначе воздействуют на текущее местонахождение роя, т.е. одна стратегия может перемещать весь рой по прямой линии, получить набор точек и перемещать также рой к каждой по прямым, другая же может манипулировать роем путём создания траекторий на основе сплайнов. Также существует третья стратегия, выраженная как параметрическая функция построения винтовой линии.

\section{4. Симуляционные тесты}

Предложенный алгоритм группового контроля был протестирован в программной симуляции Gazebo (рис. 1) позволяощей моделировать среду, модели роботов и сенсоры. Осповным преимуществом данного программного симулятора является возможность интеграции различных платформ. Симуляция работы полетного контроллера и создание точной виртуальной копии реальных квадрокоптеров с идентичными характеристиками позволяет апробировать результаты в условиях, приближенных к реальности.

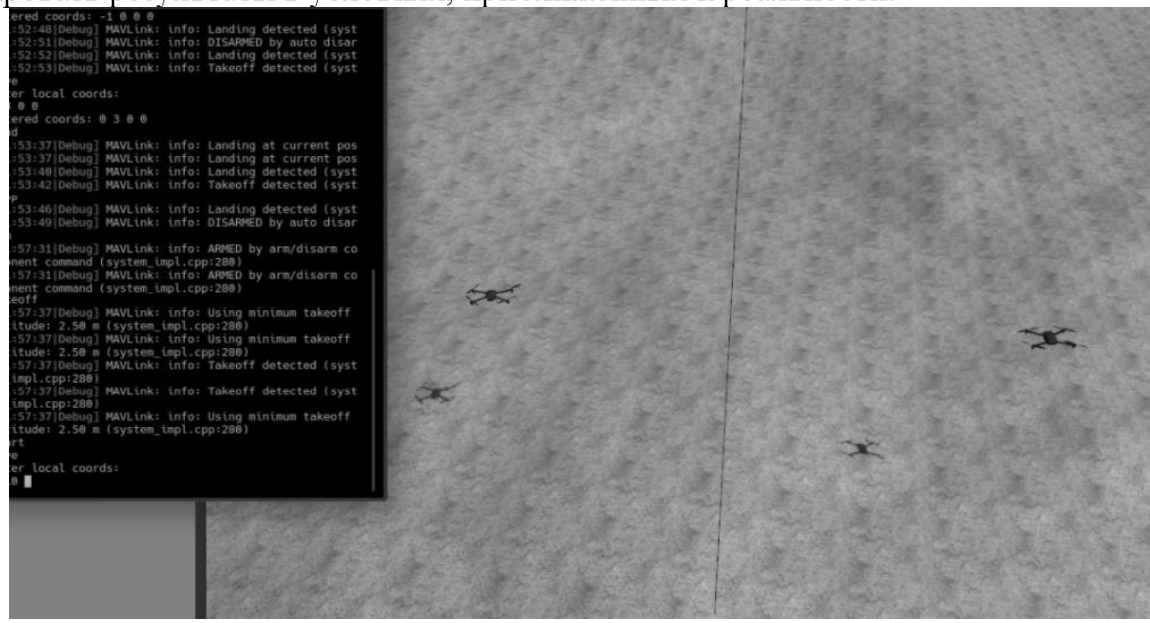

Рис. 1. Тестовый полет в Gazebo симуляции

Выводы/Заключение. В данной статье было представлено программное обеспечение, позволяющее контролировать группу квадрокоптеров с установленными полетными контроллерами Pixhawk.

В основе алгоритма лежит стратегия виртуального лидера. Программное обеспечение было разработано в программной среде $\mathrm{C}++$. Для запуска использовалась операционная система Ubuntu 19.10.

Было выполнено 10 симуляционных полетов. Каждый из тестов выполнялся с разной стартовой позицией GPS. Был определен сценарий согласно которому квадрокоптеры выполняли взлет на высоту 2,5 метров, 
занимали целевые позиции в воздухе путем построения в линию и далее двигались к задаваемым для них точкам с жестким сохранением строя (рис. 1).

В ближайшем будущем планируется модернизация программного обеспечения путем добавления дополнительных сенсоров на квадрокоптеры и реализация возможности обхода препятствий в не моделированных средах.

\section{ЛИТЕРА ТУРА}

1. R. Abbas and Q Wu. Improved leader follower formation controller for multiple quadrotors based AFSA. Telcomnika 2015, 13 (1), pages 85-92.

2. R. Olfati-Saber and R. M. Murray, Distributed cooperative control of multiple vehicle formations using structural potential functions, in Proceedings of the 15th World Congress of the International Federation of Automatic Control (IFAC '02), 2002, pp. 242-248

3. C. Moeslinger, T. Schmickl, K. Crailsheim A minimalist flocking algorithm for swarm robots, European Conference on Artificial Life: 2009, pages 375-382.

4. N. Metni and T. Hamel, "Visual tracking control of aerial robotic systems with adaptive depth estimation," International Journal of Control, Automation, and Systems, vol. 5, no. 1, 2007, pp. 51-60.

5. A. Mokhtari and A. Benallegue, "Dynamic feedback controller of Euler angles and wind parameters estimation for a quadrotor unmanned aerial vehicle," Proc. of the IEEE International Conference on Robotics and Automation, 2004, pp. 23592366 .

6. Blakelock, J.H., 1991. Automatic Control of Aircraft and Missiles 2nd ed., Wiley-Interscience.

7. Bouabdallah, S., Murrieri, P. \& Siegwart, R. Towards Autonomous Indoor Micro VTOL. Autonomous Robots, 18(2), 2005.

8. Castillo, P., Lozano, R. \& Dzul, A., 2005. Stabilization of a mini rotorcraft with four rotors. Control Systems, IEEE, 25(6), p.45- 55 .

9. De Lellis, Marcelo. Modeling, Identification, and Control of a quadrotor Aircraft. Czech Technical University in Prague, 2011.

10. URL: https://docs.px4.io/v1.9.0/en/flight_controller/pixracer.html (дата обращения 06.05.2020).

\section{OБ ABTOPAX | ABOUT AUTHORS}

Самойлов Филипп Владимирович, аспирант кафедры информационных систем и технологий, Институт информационных технологий и телекоммуникаций ФГАОУ ВО «Северо-Кавказский федеральный университет», г. Ставрополь, E-mail: Sam192@yandex.ru, Тел: + 7-918-747-21-70

Samoilov Philipp Vladimirovich, Graduate student, Chair of Information Systems and Technologies, Institute of Information Technologies and Telecommunications FSAEI HE "North-Caucasus Federal University", Stavropol, E-mail: Sam192@yandex.ru, +7-918-747-21-70

Ганьшин Константин Юрьевич, аспирант кафедры информационшых систем и технологий, Институт информационшых технологий и телекоммуникаций ФГАОУ ВО «Северо-Кавказский федеральный университет», г. Ставрополь, E-mail: magnuskos@gmail.com, Тел: + 7-918-799-05-50

Ganshin Konstantit Yuryevich, Graduate student, Chair of Information Systems and Technologies, Institute of Information Technologies and Telecommunications FSAEI HE "North-Caucasus Federal University", Stavropol, E-mail: magnuskos@gmail.com,+7-918-799-05-50.

Мезенцева Оксана Станис.тавовна, кандидат физико-математических наук, Профессор кафедры информационных систем и технологий, Институт информационных технологий и телекоммуникаций ФГАОУ ВО «Северо-Кавказский федеральный университет», г. Ставрополь,

E-mail: omezentceva@ncfu.ru, 8(8652)94-54-75

Mezentseva Oksana Stanislavovna, Candidate of physical-mathematical sciences, Professor of the Depart-

ment

of Information Systems and Technologies, Institute of Information Technologies and Telecommunications FSAEI HE "North-Caucasus Federal University", StavropolE-mail: omezentceva@ncfu.ru, 8(8652) 94-54-75

Винокурский Дмитрий Лсошидовит, кандидат физико-математических наук, доцент кафедры информационных систем и технологий, Институт информационных технологий и телекоммуникаций ФГАОУ ВО «Северо-Кавказский федеральный университет», г. Ставрополь,

E-mail: dlvinokursky@gmail.com, Тел: + 7-918-877-39-23

Vinokursky Dmitry Leonidovich, Candidate of physical-mathematical sciences, docent of Chair of Information Systems and Technologies, Institute of Information Technologies and Telecommunications FSAEI HE "North-Caucasus Federal University", Stavropol, E-mail: dlvinokursky@gmail.com, $+7-918-877-39-23$ 